\title{
Saúde e pesquisas domiciliares no Brasil e Inglaterra: a Pesquisa Nacional de Saúde e a Health Survey for England
}

\author{
Health and household surveys in Brazil and England: \\ The National Health Survey and the Health Survey for England
}

César Marques (https://orcid.org/0000-0003-4935-112X) ${ }^{1}$

Igor Cavallini Johansen (https://orcid.org/0000-0002-5360-3740) ${ }^{2}$

${ }^{1}$ Escola Nacional de Ciências Estatísticas. R. André Cavalcanti 106, Centro. 20231-050 Rio de Janeiro RJ Brasil. cesar.m.silva@ibge.gov.br ${ }^{2}$ Núcleo de Estudos e Pesquisas Ambientais, Universidade Estadual de Campinas. Campinas SP Brasil.

\begin{abstract}
This paper aims to analyze the characteristics of national health surveys conducted in Brazil and England by their respective Statistics institutes. For Brazil, the National Health Survey (PNS) was considered, and the Health Survey for England (HSE) for England. To this end, we show a preliminary overview of the different population profiles of the two countries. Then, a brief historical background is presented, including the common themes that are addressed in the PNS and HSE that favor comparative analyses. Finally, we compared, for example, the inequalities in access to and use of Brazilian and English health services. The results show several possibilities for comparative analysis on topics such as health perception, tobacco use, alcohol consumption, diabetes, and hypertension. However, the need to consider the specificities of the population profile of each country and the methodological characteristics of the surveys is emphasized.
\end{abstract}

Key words Public health, Health surveys, Comparative study, Brazil, England
Resumo O objetivo do artigo é analisar as características das pesquisas nacionais de saúde realizadas no Brasil e na Inglaterra pelos seus respectivos institutos de Estatística. No Brasil foi considerada a Pesquisa Nacional de Saúde (PNS) e na Inglaterra, a Health Survey for England (HSE). Para tanto, apresenta-se inicialmente um panorama sobre os distintos perfis populacionais dos países. Passa-se, então, a um breve histórico das pesquisas, evidenciando inclusive as temáticas comuns que são abordadas na PNS e na HSE que favorecem análises comparativas. Por fim, apresenta-se uma comparação, a título de exemplo, acerca das desigualdades no acesso e na utilização dos serviços de saúde no Brasil e na Inglaterra. Os resultados evidenciam inúmeras possiblidades de análises comparativas em temáticas como percepção de saúde, tabagismo, consumo de bebidas alcoólicas, diabetes e hipertensão. Entretanto, salienta-se a necessidade de atentar para especificidades do perfil populacional de cada país e às características metodológicas das pesquisas.

Palavras-chave Saúde pública, Inquéritos de saúde, Estudo comparativo, Brasil, Inglaterra 


\section{Introdução}

A realização de inquéritos nacionais de saúde tem sido constante desde ao menos a década de 1960 em países de alta renda, sendo mais recente em países de renda média e baixa. Tais avanços ocorreram no contexto de ampliação da geração de informações que subsidiam o planejamento, monitoramento e avaliação de políticas públicas em saúde. Essas atendem a múltiplos propósitos, passando pelo levantamento do perfil de saúde da população, distribuição dos fatores de risco, estimação dos condicionantes socioambientais da saúde, avaliação dos sistemas de saúde e estimação de iniquidades na população analisada ${ }^{1}$. Juntamente às demais fontes de informação e registros administrativos, tais pesquisas são fundamentais para o conhecimento do estado e evolução da saúde de populações. Contudo, tendo em vista os custos e complexidade dessas pesquisas, sua periodicidade raramente é anual.

Nesse contexto, o Brasil realizou seu primeiro inquérito da Pesquisa Nacional de Saúde (PNS) em 2013, com a segunda aplicação em 2019. A Inglaterra, por sua vez, realiza pesquisas anuais focadas na saúde da população desde a década de 1970 com os inquéritos do General Health Survey (GHS) (a pesquisa foi renomeada para General Lifestyle Survey em 2008), e a Health Survey for England (HSE), iniciada em 1991.

Neste artigo, temos como objetivo analisar as características das pesquisas nacionais de saúde realizadas no Brasil e na Inglaterra pelos seus respectivos institutos de Estatística. No Brasil será considerada a PNS, conduzida pelo IBGE, e, na Inglaterra, a HSE, coordenada conjuntamente pelo NatCen Social Research e a University College London. Para tal, primeiramente são apresentadas as principais questões de saúde nos países a partir de aspectos da dinâmica demográfica e da morbimortalidade nos dois países. Em um segundo momento e com base nessa análise, consideramos comparativamente quais são as principais características da PNS e da HSE, indicando os temas abordados e as respectivas possibilidades de análise comparativa. Por fim são feitas comparações de indicadores selecionados que sejam representativos das desigualdades no acesso e na utilização dos serviços de saúde a partir dos inquéritos nacionais domiciliares.

\section{População e saúde: Brasil e Inglaterra}

As diferentes situações de saúde entre países podem ser consideradas como condicionadas por uma série de processos socioeconômicos e históricos. Para o caso em questão, na comparação Brasil-Inglaterra, destacam-se as mudanças demográficas e as atuais tendências de saúde.

Em termos populacionais, o processo de transição demográfica, dado pelas mudanças de um regime em que prevalecem altas taxas de mortalidade e natalidade para um em que ambas as taxas sejam baixas, é considerado como um dos fenômenos centrais da mudança populacional na sociedade moderna. Esse pode ser notado como um processo amplo, intrinsecamente interligado com a modernização da sociedade e com a urbanização ${ }^{2,3}$.

No Brasil, esse processo ocorreu ao longo do século XX, acelerando-se a partir dos anos 1950 em conjunção com um rápido processo de urbanização, que marcou a mudança de um país até então fundamentalmente rural para majoritariamente urbano. Nesse contexto, mortalidade e fecundidade apresentaram quedas expressivas. Entre 1950 e 2010, a taxa de fecundidade total (TFT) - que compreende o número médio de filhos que uma mulher terá ao longo de sua vida reprodutiva - declinou rapidamente, passando de 6,2 para 1,9. A taxa de mortalidade infantil (TMI), por sua vez, denota o risco de morrer antes do primeiro ano de vida, sendo importante indicador de desenvolvimento socioeconômico. A TMI no Brasil caiu de 135 para 16,2 por mil nascidos vivos entre 1950 e 2020, enquanto a expectativa de vida ao nascer subiu de 45,5 para 73,5 anos. Durante o mesmo período, a razão de dependência, ou seja, o quociente entre a população definida como economicamente "dependente" (os menores de 15 anos de idade e os de 60 anos e mais) sobre o segmento etário potencialmente produtivo (entre 15 e 59 anos de idade), mais que dobrou, de 8,0\% no início do período e $16,6 \%$ ao final ${ }^{4}$. Como se pode observar, houve uma rápida transição na estrutura etária, gerando pressões nos sistemas previdenciário e de saúde. Este último passa a atentar para as demandas relacionadas ao envelhecimento populacional, com serviços voltados à prevenção de doenças crônicas, por exemplo ${ }^{5}$. Vale ressaltar, contudo, que dada a dimensão territorial do país e suas desigualdades socioeconômicas inter e intra-regionais, a transição demográfica ocorre em ritmos diferentes em cada localidade, com populações e regiões mais pobres apresentando TFTs e níveis de mortalidade relativamente elevados mesmo em momentos mais recentes ${ }^{6,7}$.

Já na Inglaterra, a transição demográfica tem seu início geralmente datado ainda no final do 
século XIX, com as quedas paulatinas da mortalidade e fecundidade ${ }^{8,9}$. As estimativas para fecundidade indicam que essas passaram de aproximadamente 6,0 filhos por mulher durante a década de 1870 para alcançar o nível de reposição já na década de $1930^{10}$. O nível de reposição compreende o limiar mínimo de 2,1 filhos por mulher para que a população permaneça numericamente estável. Entre 1930 e 1970 as TFTs na Inglaterra oscilaram em decorrência de mudanças sociais e econômicas significativas vivenciadas no período, que compreende a 2a Guerra Mundial (1939-1945) e a recuperação dos países envolvidos no momento seguinte. Os níveis mais baixos foram vistos durante a guerra, com a TFT de 1,7 em 1941. Em 1947 esse indicador já alcançava 2,7 filhos por mulher e, em 1964, 2,9. A fecundidade apresentou a partir de então uma queda significativa, tendo oscilado entre 1,6 e 1,9 durante as décadas de 1980 e $2010^{11}$.

A mortalidade no país também apresentou quedas a partir da segunda metade do século XIX, de modo que a expectativa de vida ao nascer, de 41 anos em 1841, passou para aproximadamente 50 anos no início do século XX e cresceu intensamente na primeira metade desse século, chegando a 71 anos na década de 1960. Tais avanços ocorreram majoritariamente pela queda na mortalidade infantil e na infância ${ }^{12}$. Já na segunda metade do século XX, com avanços nos tratamentos médicos e cuidados da saúde da população adulta e idosa, houve queda das taxas de mortalidade de grupos etários com maior idade e aumento da longevidade, com a expectativa de vida alcançando 81 anos em 2010

As mudanças demográficas ocorridas na Inglaterra estão amplamente documentadas e discutidas, tendo-se apontado como fatores explicativos, por exemplo, mudanças sociais impactando na transição da fecundidade ${ }^{9,13}$ e avanços sanitários com seus efeitos sobre a queda na ocorrência de doenças infectocontagiosas ainda no século XIX ${ }^{14,15}$.

Diante de processos históricos distintos que, por sua vez, apresentam impactos sobre a dinâmica populacional e, consequentemente, de saúde, as estruturas etárias brasileira e inglesa apresentam atualmente diferenças significativas, como evidenciado na Figura 1.

Embora ambos os países se encontrem em estágios avançados da transição da fecundidade, suas estruturas etárias indicam claramente as importantes diferenças nas velocidades e tempos da transição demográfica, com a distribuição etária da Inglaterra mais envelhecida, apresentando proporção significativamente maior de idosos e menor de crianças em relação ao Brasil. Além disso, a distribuição dos grupos etários proporcionalmente ao total também é mais equânime no caso do país europeu, enquanto o Brasil apresenta preponderância dos grupos populacionais entre 10 e 29 anos.

Contudo, se durante os anos 2000-2010 os dois países apresentaram ganhos na expectativa de vida (embora de forma mais lenta em relação ao passado recente), o caso inglês tem apresentado tendência à estagnação. Considerando o Reino Unido como um todo, houve, para a população jovem e adulta (entre 15 e 54 anos), reversão da tendência da queda da mortalidade e, para a população com 90 anos e mais, constatou-se estabilidade com leve ascensão ${ }^{16}$. Mesmo em comparação com outros países desenvolvidos, a Inglaterra apresentou uma evolução mais lenta da expectativa de vida na década de 2010. Tal platô da expectativa de vida apresenta diferenciais de idade, sexo e grupo social, com causas relacionadas às políticas de austeridade fiscal que limitam a qualidade e o acesso aos serviços de saúde no país ${ }^{17,18}$.

$\mathrm{O}$ atual momento de saúde na Inglaterra tem se caracterizado também pelo aumento da mortalidade de idosos (90 anos e mais) em função de doenças mentais e comportamentais ${ }^{16}$; o crescimento da pobreza infantil e reversão da queda da mortalidade infantil especialmente em áreas mais pobres $^{18}$; redução da velocidade de queda das taxas de mortalidade específicas de doenças circulatórias ${ }^{12}$; aumento de mortes causadas por overdoses de drogas e álcool e de doenças relacionadas ao alcoolismo e suicídios ${ }^{19}$; além do crescimento da diferença da expectativa de vida entre mais ricos e mais pobres ${ }^{20}$.

Por sua vez, o Brasil passou, ao longo do século XX, por uma transição epidemiológica considerada como incompleta. A transição epidemiológica clássica, proposta por Omran ${ }^{21}$, previa a paulatina mudança no padrão de morbimortalidade de doenças infecciosas e parasitárias para maiores incidências e prevalência de doenças crônico-degenerativas e causas externas. Prata ${ }^{22} \mathrm{e}$ Araújo $^{23}$, analisando o caso brasileiro, apontam para o crescimento de doenças crônico-degenerativas, mas com a manutenção da ocorrência em níveis elevados das enfermidades infecciosas e parasitárias (como dengue e malária) que, em teoria, deveriam ter sido superadas. Tem-se ainda o crescimento da importância de óbitos relacionados às causas externas, principalmente em acidentes de trânsito e homicídios entre jovens do sexo masculino ${ }^{24,25}$. 
A

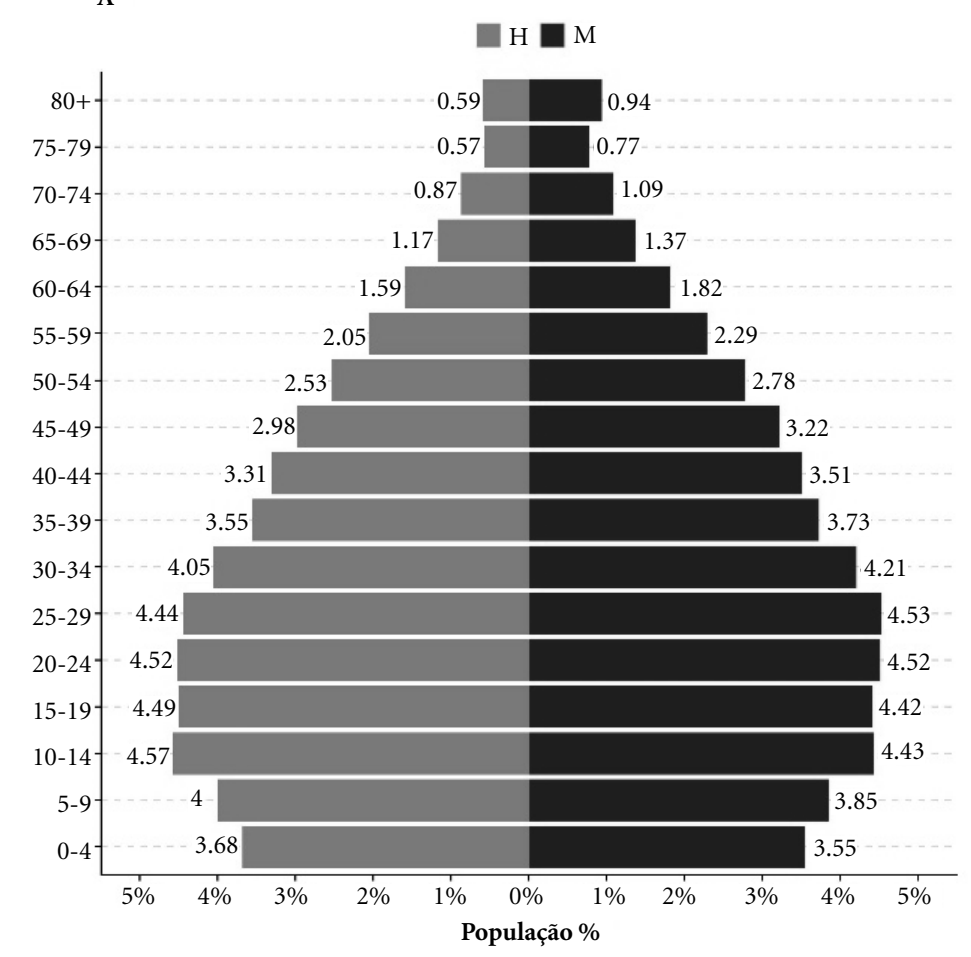

B

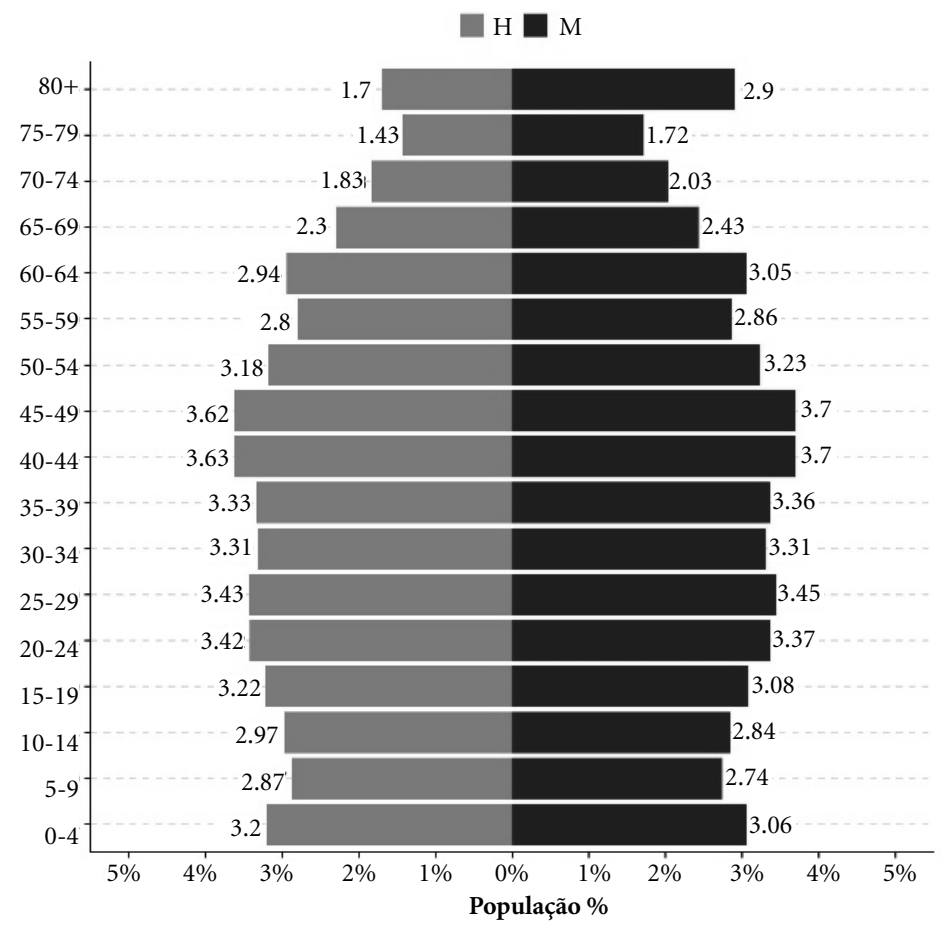

Figura 1. Estrutura etária por sexo e grupos quinquenais de idade, Brasil (A) e Inglaterra (B) - 2010 e 2011, respectivamente.

Fonte: A: IBGE - Censo Demográfico 2010. B: Population Census, Office for National Statistics, 2011. 
Aos aspectos anteriores, soma-se ainda como fator agravante na recente dinâmica de morbimortalidade do país o período de crise econômica atravessado durante a segunda década do século XXI. Nesse contexto, paralelamente à crescente taxa de desemprego, observa-se a corrosão de direitos sociais e retrocessos nas políticas de inclusão da população em piores condições socioeconômicas, além da progressiva redução dos recursos destinados à saúde pública ${ }^{26}$. Analisando o período entre 2012 e 2017 , Hone et al. ${ }^{27}$ concluíram que, considerando os gastos em saúde e os programas de proteção social, houve aumento da mortalidade em função do aumento do desemprego, principalmente nas causas relacionadas às neoplasias e doenças cardiovasculares. Tal associação foi mais expressiva em grupos específicos: na população negra, nos grupos etários entre 30 e 59 anos e entre homens. Além disso, nos municípios com gastos sociais elevados em saúde e com programas sociais não houve associação significativa entre a recessão econômica e o excesso de mortalidade.

A Tabela 1 apresenta uma análise comparativa dos panoramas sociodemográfico e de saúde de ambos os países em análise.

Dentre os indicadores apresentados, vale atentar a diferente dimensão geográfica dos países, pois enquanto o Brasil possui mais de 8,5 milhões de $\mathrm{km}^{2}$, a Inglaterra apresenta menos de $131 \mathrm{mil} \mathrm{km}^{2}$. Esse fato por si só já apresenta implicações significativas sobre o desafio de garantir a viabilidade de sistemas nacionais de saúde pública que abarquem toda sua população, além de que, no caso do Brasil, à extensão territorial adiciona-se o isolamento de parte da população em localidades de difícil acesso, como é o caso de comunidades ribeirinhas e populações indígenas isoladas na Amazônia ${ }^{39}$. Outro desafio é o volume populacional, já que a população brasileira compreende cerca de quatro vezes o número de habitantes daquele país europeu. Por outro lado, como já se mencionou, processos históricos e demográficos distintos culminam no fato de que os indicadores socioeconômicos são muito mais favoráveis na Inglaterra em comparação ao Brasil. As diferenças nesses indicadores, por sua vez, apresentam reflexos sobre a dinâmica de mortalidade nesses países. Assim, enquanto a Inglaterra apresenta taxas de mortalidades mais elevadas que o Brasil por doenças cardiovasculares, neoplasias, problemas neurológicos e doenças respiratórias; o contrário ocorre com diabetes e doenças renais, HIV/Aids, doenças negligenciadas, deficiências nutricionais e violência interpessoal, questões de saúde pública visivelmente mais agudas no Brasil.

\section{Brasil: Pesquisa Nacional de Saúde (PNS)}

As estatísticas públicas de saúde no Brasil são compostas por um conjunto de pesquisas e levantamentos, incluindo as domiciliares e registros administrativos. Do ponto de vista dos registros, alguns dos fundamentais na área da saúde são os sistemas de estatísticas vitais, como o Sistema de Informações sobre Mortalidade (SIM) e o Sistema de Informações sobre Nascidos Vivos (Sinasc); as estatísticas sobre doenças e agravos, com o Sistema de Informações sobre Agravos de Notificação (Sinan), e informações sobre internações hospitalares, com o Sistema de Informação Hospitalar do Sistema Único de Saúde (SIHSUS). Tais registros são geridos pelo Ministério da Saúde.

Já as pesquisas e levantamentos sobre saúde incluem a Pesquisa de Assistência Médico-Sanitária, que investiga os estabelecimentos públicos e privados em todo o país que prestam assistência à saúde individual ou coletiva e teve a última edição em 2009; as Estadics e Munics, realizadas junto aos governos estaduais e municipais com coleta de informações sobre a gestão, inclusive no campo da saúde (última edição realizada em 2018); a Pesquisa Nacional de Saúde do Escolar (PeNSE,) realizada em 2009, 2012 e 2015 visando investigar os fatores de risco e proteção à saúde de adolescentes, e a Pesquisa Nacional de Saúde (PNS), que será aqui analisada. Vale ressaltar, contudo, que a situação de saúde do país como um todo requer a análise do conjunto das pesquisas e não é objetivo desse artigo.

Historicamente, a PNS remonta às pesquisas domiciliares que retrataram o sistema de saúde no país ainda na década de 1980, quando, em 1981 e 1986, a Pesquisa Nacional por Amostra de Domicílios (PNAD) incluiu suplementos sobre a saúde. A pesquisa, mais ampla na sua primeira aplicação ${ }^{40}$, compreendeu a coleta de dados via questionário, a aferição de medidas antropométricas e a coleta de sangue e urina para análise laboratorial. O suplemento saúde voltou a ser aplicado somente em 1998, quando foi incorporado em um período regular de cinco anos, com investigação realizada também em 2003 e 2008.

A primeira realização da PNS ocorreu em 2013 em uma iniciativa conjunta do Instituto Brasileiro de Geografia e Estatística (IBGE) e Ministério da Saúde para investigar as condições de saúde e estilos de vida da população no Brasil a 
Tabela 1. Indicadores sociodemográficos e de saúde, Brasil e Inglaterra, em anos selecionados.

\begin{tabular}{|c|c|c|}
\hline Indicadores & Brasil & Inglaterra \\
\hline Area, $\mathrm{km}^{2}$ & 8.538 .000 & 130.427 \\
\hline População total & $210.147 .125^{\mathrm{a}}$ & $56.286 .961^{\mathrm{b}}$ \\
\hline Coeficiente de Gini, 2018 e 2016, respectivamente ${ }^{c}$ & 0,54 & $0,35^{\mathrm{d}}$ \\
\hline Nível de renda ${ }^{e}$ & Média alta & Alta $^{\mathrm{d}}$ \\
\hline PIB per capita, em dólares americanos ${ }^{e}$ & 8.717 & 42.330 \\
\hline $\begin{array}{l}\text { População com menos de } \$ 1.90 / \text { dia, \%, } 2018 \text { e } 2016 \text {, respectivamente, em } \\
\text { dólares americanos }{ }^{\mathrm{f}}\end{array}$ & 4,4 & $0,2^{\mathrm{d}}$ \\
\hline População vivendo em áreas urbanas, $2020, \%^{\mathrm{g}}$ & 87,1 & $83,9^{d}$ \\
\hline Expectativa de vida ao nascer, anos, homens $(2015-2020)^{\mathrm{h}}$ & 71,90 & $79,37^{\mathrm{d}}$ \\
\hline Expectativa de vida ao nascer, anos, mulheres $(2015-2020)^{\mathrm{h}}$ & 79,27 & $82,90^{\mathrm{d}}$ \\
\hline $\begin{array}{l}\text { Taxa de mortalidade de crianças menores de } 5 \text { anos, por } 1.000 \text { nascidos } \\
\text { vivos, } 2019^{i}\end{array}$ & 13,9 & $4,3^{\mathrm{d}}$ \\
\hline Pessoas com saneamento adequado, $2017, \%^{j}$ & 97,8 & $49,3^{\mathrm{d}}$ \\
\hline Taxa de fecundidade total ${ }^{\mathrm{k}}$ & 1,8 & 1,8 \\
\hline Anos de escolaridade ${ }^{k}$ & 7,9 & 12,9 \\
\hline \multicolumn{3}{|l|}{ Taxa de mortalidade, por causas selecionadas, por 1.000 hab., $2019^{1}$} \\
\hline Doenças cardiovasculares & 183,6 & 270,9 \\
\hline Neoplasias & 122,8 & 283,8 \\
\hline Problemas neurológicos & 32,7 & 70,1 \\
\hline Infecções respiratórias e tuberculose & 43,5 & 62,3 \\
\hline Doenças respiratórias crônicas & 35,3 & 74,7 \\
\hline Diabetes e doenças renais & 49,7 & 20,2 \\
\hline HIV/AIDS e doenças sexualmente transmissíveis & 7,4 & 0,5 \\
\hline Doenças negligenciadas e malária & 4,2 & 0,0 \\
\hline Deficiências nutricionais & 3,8 & 0,2 \\
\hline Automutilação e violência interpessoal & 37,2 & 8,3 \\
\hline
\end{tabular}

partir de uma pesquisa domiciliar incorporada ao Sistema Integrado de Pesquisas Domiciliares (SIPD), com uma periodicidade prevista de cinco anos ${ }^{41,42}$. De modo geral, a PNS é caracterizada como uma pesquisa domiciliar que permite a investigação sobre as condições de saúde da população, a avaliação, o uso e o acesso do sistema nacional de saúde; e a vigilância de doenças e agravos de saúde e fatores de risco associados ${ }^{43}$.

A amostra da PNS é feita por conglomerado em três estágios de seleção, sendo uma subamostra da Amostra Mestra do SIPD (que por sua vez tem como base o Censo Demográfico 2010). A Amostra Mestra é dada por um conjunto de unidades primárias de amostragem (UPAs), representadas por setores censitários ou um conjunto de setores (a depender da quantidade de domicílios nos mesmos). A estratificação das UPAs ocorre segundo quatro critérios: administrativo (UF, capital, restante da Região Metropolitana
(RM) ou Região Integrada de Desenvolvimento Econômico (RIDE) e restante da UF); geográfico (subdivisão de capitais e munícipios maiores em estratos), de situação (urbano e rural), e estatístico (os estratos são selecionados a partir da homogeneidade, considerando rendimento e totais de domicílios) ${ }^{43,44}$.

No primeiro estágio, as UPAs são selecionadas por amostragem aleatória simples (AAS) na amostra de um trimestre da Amostra Mestra. No segundo, via AAS, é selecionado um número fixo de domicílios particulares permanentes seguindo o Cadastro Nacional de Endereços para Fins Estatísticos (CNEFE). Como próximo passo, em cada domicílio é selecionado um residente com ao menos 15 anos de idade via AAS, que responde ao questionário individual. Assim, os dados relacionados aos domicílios são feitos a partir de dois estágios de seleção, e o dos moradores com três estágios. Por fim, o cálculo do tama- 


\section{Inglaterra: Health Survey for England} (HSE)

As pesquisas domiciliares sobre saúde no Reino Unido, realizadas periodicamente desde a década de 1970 com a aplicação do General Household Survey (GHS), incluem não só levantamentos amostrais como também censitários, isto é, que englobam toda a população. No âmbito das pesquisas amostrais, a HSE, iniciada em 1991 e com periodicidade anual, é uma das principais fontes de estatísticas certificadas como oficiais sobre saúde na Inglaterra. É realizada pela Health Surveys Unit do NatCen Social Research em parceria com o Research Department of Epidemiology and Public Health da University College London 45.

A pesquisa inclui a coleta anual de indicadores de saúde, psicossociais, sobre consumo de cigarro e álcool, uso de serviços de saúde, de medicamentos e coleta de medidas antropométricas sobre obesidade e pressão arterial, dentre outras. Além disso, são realizados suplementos específicos (que variam anualmente), que cobrem regiões, temas ou grupos populacionais ou étnicos específicos. A HSE é aplicada nos domicílios particulares, com cobertura de informações da população adulta com 16 anos ou mais (até 1995), e de toda a população (a partir de 1996). É representativa para essa população no país como um todo e desagregada para regiões administrativas $^{45}$.

Em 2019, assim como anteriormente, a metodologia da seleção dos entrevistados seguiu amostragem probabilística estratificada, sendo a base da amostra o cadastro de endereços postais. Nesse ano, no primeiro estágio foi realizada a seleção de uma amostra aleatória de unidades primárias de amostragem (UPAs) em setores de código postal, gerando 534 UPAs. Posteriormente, para cada UPA são selecionados 18 endereços, resultando em um total de 9.612 endereços (dos quais $90 \%$ efetivamente continham domicílios particulares). Os domicílios visitados tiveram todos os moradores entrevistados, considerando um limite de até dez entrevistas para a popula- ção com 16 anos ou mais, duas entrevistas para aqueles com até 12 anos e duas entrevistas para os grupos entre 13 e 15 anos. A realização de coleta de dados mais amplos sobre as condições de saúde, com visita de enfermeiros, foi realizada em uma seleção aleatória de 16 endereços em cada UPA. As taxas de resposta por domicílio foram de $60 \%$, considerando que ao menos um morador elegível foi entrevistado, e de $47 \%$ para realização de entrevistas com todos os moradores elegíveis. Ao todo, em 2019 a amostra teve 8.205 adultos e 2.095 crianças de até 15 anos, sendo que 4.947 adultos e 1.169 crianças tiveram também coleta por enfermeiros ${ }^{45}$.

PNS e HSE: análise comparativa das metodologias e de indicadores selecionados

A PNS e a HSE são fundamentais para os sistemas de estatísticas sobre saúde no Brasil e Inglaterra, respectivamente. Contudo, os históricos e execução variaram grandemente. Na Inglaterra, a pesquisa provê dados anuais desde 1991, enquanto no Brasil a PNS é realizada apenas desde 2013 (ou 1998, considerando o suplemento Saúde da PNAD), tendo a mais recente edição ocorrido em 2019. Por outro lado, no Brasil o tamanho da amostra propicia uma desagregação espacial no nível de Unidades de Federação e municípios das capitais, enquanto a HSE é restrita ao nível do país e regiões administrativas. A comparação das principais características metodológicas das pesquisas é apresentada no Quadro 1.

A comparação entre os temas pode ser observada no Quadro 2. De modo geral, tanto PNS como na HSE abordam a percepção do estado de saúde, tabagismo, consumo de bebidas alcoólicas, consumo de frutas, medidas antropométricas (altura, peso e circunferência da cintura), hipertensão arterial, colesterol alto, problemas renais e problemas respiratórios. Especificamente na Inglaterra, é relevante notar a presença de questões inseridas mais recentemente, mas que permanecem ininterruptamente desde então: planos de saúde, desde 2009, e orientação sexual, desde 2011. O Brasil também inseriu questão sobre orientação sexual na edição de 2019, além de manter perguntas que abarquem fenômenos importantes, mas com dados relativamente escassos no país, como sobre saúde materna.

Existem, portanto, diversas possibilidades de análises comparativas entre temáticas de saúde no Brasil e Inglaterra utilizando a PNS e HSE. Ambas trazem tendências já consolidadas das condições de saúde e permitem análises sobre 
Quadro 1. Comparação metodológica entre as pesquisas: PNS e HSE.

\begin{tabular}{|c|c|c|}
\hline & PNS $^{\text {a }}$ & HSE $^{b}$ \\
\hline Anos & 2013/2019 (2 edições) & 1991-2019 (29 edições) \\
\hline Idades abarcadas & Todas & Todas (desde 1996) \\
\hline Respondentes & $\begin{array}{l}\text { Residentes } 15 \text { anos aleatoriamente } \\
\text { escolhidos entre os elegíveis no domicílio }\end{array}$ & $\begin{array}{l}\text { Residentes } 16 \text { anos aleatoriamente escolhidos } \\
\text { entre os elegíveis no domicílio }\end{array}$ \\
\hline Plano amostral & $\begin{array}{l}\text { Amostragem conglomerada em três } \\
\text { estágios: setores censitários ou conjunto de } \\
\text { setores; domicílios; e respondentes com } 15 \\
\text { anos ou mais (terceiro estágio). Total: cerca } \\
\text { de } 290 \text { mil pessoas em } 80 \text { mil domicílios. }\end{array}$ & $\begin{array}{l}\text { Amostragem conglomerada em três estágios: } \\
\text { áreas postais; domicílios; e respondentes com } \\
16 \text { anos ou mais (terceiro estágio). Total: Cerca } \\
10 \text { mil pessoas em } 4 \text { mil domicílios. }\end{array}$ \\
\hline $\begin{array}{l}\text { Excluídos da } \\
\text { amostra }\end{array}$ & $\begin{array}{l}\text { Populações de aldeias indígenas e } \\
\text { institucionalizados (em quartéis, bases } \\
\text { militares, alojamentos, acampamentos, } \\
\text { embarcações, penitenciárias, colônias } \\
\text { penais, presídios, cadeias, asilos, orfanatos, } \\
\text { conventos e hospitais) }\end{array}$ & $\begin{array}{l}\text { Institucionalizados (domicílios não } \\
\text { particulares) }\end{array}$ \\
\hline $\begin{array}{l}\text { Nível geográfico } \\
\text { de desagregação }\end{array}$ & $\begin{array}{l}\text { Brasil - Grandes Regiões - Unidades da } \\
\text { Federação - Municípios das capitais }\end{array}$ & Inglaterra - Regiões administrativas. \\
\hline
\end{tabular}

as mudanças sociais subjacentes. Em relação à características mais perenes, os dados relativos a 2019 mostram, por exemplo, que a proporção de mulheres que realizam consultas médicas é de 78,3\% no país europeu e $82,3 \%$ no latino-americano, maior do que a de homens (respectivamente $57,3 \%$ e 69,4\%), para consultas nos 12 meses anteriores à data de entrevista). Por outro lado, considerando esse indicador por status socioeconômico, na Inglaterra os percentuais são próximos: entre aqueles em piores condições socioeconômicas, $77,9 \%$ buscaram atendimento no período, enquanto o mesmo ocorreu para $75,0 \%$ daqueles em menor vulnerabilidade social. No Brasil, por outro lado, enquanto 67,6\% do grupo com até um quarto de salário mínimo buscaram atendimento médico, 89,6\% daqueles com proventos acima de cinco salários mínimos indicaram ter realizado consulta médica no mesmo período. Tem-se aí, portanto, uma diferença muito mais expressiva comparativamente ao caso inglês, apontando para o maior acesso aos serviços de saúde pela população afluente em relação àquela socialmente vulnerável no Brasil.

Em relação à razão para visitar o médico, as perguntas se diferenciam em termos de possibilidades de respostas e período considerado. $\mathrm{Na}$ Inglaterra foram declarados como principais motivos para visita médica no período de referência: problema físico de saúde $(83,7 \%)$; problema mental, nervoso ou emocional (5,2\%); e ambos os motivos $(11,1 \%)$. Já no Brasil, o período de referência considera as duas últimas semanas, e os principais motivos de consulta médica foram: doença ou tratamento de doença $(48,2 \%)$; vacinação, prevenção, check-up médico ou acompanhamento com outro profissional de saúde $(25,1 \%)$; exame complementar de diagnóstico $(10,2 \%)$; consulta odontológica $(6,3 \%)$ e acidente, lesão ou fratura $(4,6 \%)$.

A diferença por renda entre os grupos (seguindo a classificação anterior) também foi significativa para outras dimensões em ambos os países: no Brasil, 30,8\% da população com 18 anos ou mais de idade no grupo de menor renda foi classificada como insuficientemente ativa e, no grupo de maior renda, $48,0 \%$ da população. Na Inglaterra os percentuais foram de 31,7\% para aqueles em áreas mais afluentes e 49,6\% dos que vivem em áreas com maior privação (dados relativos a 2016, última edição da pesquisa com mensuração da dimensão). No Brasil os diferenciais de renda também repercutem sobre a autoavaliação de saúde boa ou muito boa, que atinge a marca de $55,5 \%$ para os mais pobres e $86,4 \%$ para os mais ricos. Na Inglaterra, por sua vez, os dados de autoavaliação da saúde em 2019 não estão disponíveis por nível de privação, sendo que $74,6 \%$ da população teve autoavaliação boa e muito boa de saúde.

Tem-se, portanto, que, enquanto algumas perguntas podem ser facilmente comparadas entre Brasil e Inglaterra utilizando a PNS e a HSE, outras são elaboradas para evidenciar os prin- 
Quadro 2. Temas comuns em pelo menos uma edição da PNS e HSE.

\begin{tabular}{|c|c|c|c|c|c|c|c|c|c|c|c|c|c|c|c|c|c|c|c|c|c|c|c|c|c|c|c|c|c|}
\hline & PN & $\mathrm{JS}^{\mathrm{a}}$ & & & & & & & & & & & & & & HSE & & & & & & & & & & & & & \\
\hline $\begin{array}{c}\text { Tema/ } \\
\text { Ano }\end{array}$ & 尔 & 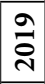 & ๙ू & 亏ั & \% & ๙ & ล & ๙̊ & बे & ¿্ণ & 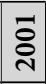 & ชั & 苂 & 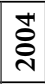 & 농 & \begin{tabular}{|l|}
\multirow{2}{*}{} \\
\end{tabular} & 命 & 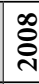 & ஓे & 응 & 공 & ก) & 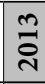 & 离 & $\frac{1}{8}$ & 웅 & 音 & $\frac{\infty}{0}$ & ) \\
\hline $\begin{array}{l}\text { Informações do } \\
\text { domicílio }\end{array}$ & $\mathrm{P}$ & $\mathrm{P}$ & $\mathrm{P}$ & $\mathrm{P}$ & $\mathrm{P}$ & $\mathrm{P}$ & $\mathrm{P}$ & $\mathrm{P}$ & $\mathrm{P}$ & $\mathrm{P}$ & $\mathrm{P}$ & $\mathrm{P}$ & $\mathrm{P}$ & $\mathrm{P}$ & $\mathrm{P}$ & $\mathrm{P}$ & $\mathrm{P}$ & $\mathrm{P}$ & $\mathrm{P}$ & $\mathrm{P}$ & $\mathrm{P}$ & $\mathrm{P}$ & $\mathrm{P}$ & $\mathrm{P}$ & $\mathrm{P}$ & $\mathrm{P}$ & $\mathrm{P}$ & $\mathrm{P}$ & $\mathrm{P}$ \\
\hline $\begin{array}{l}\text { Informações do } \\
\text { indivíduo }\end{array}$ & $\mathrm{P}$ & $\mathrm{P}$ & $\mathrm{P}$ & $\mathrm{P}$ & $\mathrm{P}$ & $\mathrm{P}$ & $\mathrm{P}$ & $\mathrm{P}$ & $\mathrm{P}$ & $\mathrm{P}$ & $\mathrm{P}$ & $\mathrm{P}$ & $\mathrm{P}$ & $P$ & $\mathrm{P}$ & $\mathrm{P}$ & $\mathrm{P}$ & $P$ & $\mathrm{P}$ & $\mathrm{P}$ & $\mathrm{P}$ & $\mathrm{P}$ & $\mathrm{P}$ & $\mathrm{P}$ & $\mathrm{P}$ & $\mathrm{P}$ & $\mathrm{P}$ & $\mathrm{P}$ & $\mathrm{P}$ \\
\hline $\begin{array}{l}\text { Benefícios } \\
\text { sociais }\end{array}$ & $\mathrm{P}$ & $\mathrm{P}$ & & & & & & & & $\mathrm{P}$ & & & & & & & & & & & & & & & & & & & \\
\hline Ocupação & $\mathrm{P}$ & $\mathrm{P}$ & & & & & & & & & & & & & & & & & & $\mathrm{P}$ & $\mathrm{P}$ & $\mathrm{P}$ & $\mathrm{P}$ & $\mathrm{P}$ & $\mathrm{P}$ & $\mathrm{P}$ & $\mathrm{P}$ & $\mathrm{P}$ & $\mathrm{P}$ \\
\hline $\begin{array}{l}\text { Percepção do } \\
\text { estado de saúde }\end{array}$ & $\mathrm{P}$ & $\mathrm{P}$ & $\mathrm{P}$ & $\mathrm{P}$ & $\mathrm{P}$ & $\mathrm{P}$ & $\mathrm{P}$ & $\mathrm{P}$ & $\mathrm{P}$ & $\mathrm{P}$ & $\mathrm{P}$ & $\mathrm{P}$ & $\mathrm{P}$ & $\mathrm{P}$ & $\mathrm{P}$ & $\mathrm{P}$ & $\mathrm{P}$ & $\mathrm{P}$ & $\mathrm{P}$ & $\mathrm{P}$ & $\mathrm{P}$ & $\mathrm{P}$ & $\mathrm{P}$ & $\mathrm{P}$ & $\mathrm{P}$ & $\mathrm{P}$ & $\mathrm{P}$ & $\mathrm{P}$ & $\mathrm{P}$ \\
\hline Tabagismo & $\mathrm{P}$ & $\mathrm{P}$ & $\mathrm{P}$ & $\mathrm{P}$ & $\mathrm{P}$ & $\mathrm{P}$ & $\mathrm{P}$ & $\mathrm{P}$ & $\mathrm{P}$ & $\mathrm{P}$ & $\mathrm{P}$ & $\mathrm{P}$ & $\mathrm{P}$ & $\mathrm{P}$ & $\mathrm{P}$ & $\mathrm{P}$ & $\mathrm{P}$ & $\mathrm{P}$ & $\mathrm{P}$ & $\mathrm{P}$ & $\mathrm{P}$ & $\mathrm{P}$ & $\mathrm{P}$ & $\mathrm{P}$ & $\mathrm{P}$ & $\mathrm{P}$ & $\mathrm{P}$ & $\mathrm{P}$ & $\mathrm{P}$ \\
\hline $\begin{array}{l}\text { Consumo } \\
\text { de bebidas } \\
\text { alcoólicas }\end{array}$ & $\mathrm{P}$ & $\mathrm{P}$ & $\mathrm{P}$ & $\mathrm{P}$ & $\mathrm{P}$ & $\mathrm{P}$ & $\mathrm{P}$ & $\mathrm{P}$ & $\mathrm{P}$ & $\mathrm{P}$ & $\mathrm{P}$ & $\mathrm{P}$ & $\mathrm{P}$ & $\mathrm{P}$ & $\mathrm{P}$ & $\mathrm{P}$ & $\mathrm{P}$ & $\mathrm{P}$ & $\mathrm{P}$ & $\mathrm{P}$ & $\mathrm{P}$ & $\mathrm{P}$ & $\mathrm{P}$ & $\mathrm{P}$ & $\mathrm{P}$ & $\mathrm{P}$ & $\mathrm{P}$ & $\mathrm{P}$ & $\mathrm{P}$ \\
\hline $\begin{array}{l}\text { Consumo de } \\
\text { frutas }\end{array}$ & $\mathrm{P}$ & $\mathrm{P}$ & $\mathrm{P}$ & $\mathrm{P}$ & $\mathrm{P}$ & $\mathrm{P}$ & $\mathrm{P}$ & $\mathrm{P}$ & $\mathrm{P}$ & $\mathrm{P}$ & $\mathrm{P}$ & $\mathrm{P}$ & $\mathrm{P}$ & $\mathrm{P}$ & $\mathrm{P}$ & $\mathrm{P}$ & $\mathrm{P}$ & $\mathrm{P}$ & $\mathrm{P}$ & $\mathrm{P}$ & $\mathrm{P}$ & $\mathrm{P}$ & $\mathrm{P}$ & $\mathrm{P}$ & $\mathrm{P}$ & $\mathrm{P}$ & $\mathrm{P}$ & $\mathrm{P}$ & $\mathrm{P}$ \\
\hline Altura & $\mathrm{P}$ & $\mathrm{P}$ & $\mathrm{P}$ & $\mathrm{P}$ & $\mathrm{P}$ & $\mathrm{P}$ & $\mathrm{P}$ & $\mathrm{P}$ & $\mathrm{P}$ & $\mathrm{P}$ & $\mathrm{P}$ & $\mathrm{P}$ & $\mathrm{P}$ & $\mathrm{P}$ & $\mathrm{P}$ & $\mathrm{P}$ & $\mathrm{P}$ & $\mathrm{P}$ & $\mathrm{P}$ & $\mathrm{P}$ & $\mathrm{P}$ & $\mathrm{P}$ & $\mathrm{P}$ & $\mathrm{P}$ & $\mathrm{P}$ & $\mathrm{P}$ & $\mathrm{P}$ & $\mathrm{P}$ & $\mathrm{P}$ \\
\hline Peso & $\mathrm{P}$ & $\mathrm{P}$ & $\mathrm{P}$ & $\mathrm{P}$ & $\mathrm{P}$ & $\mathrm{P}$ & $\mathrm{P}$ & $\mathrm{P}$ & $\mathrm{P}$ & $\mathrm{P}$ & $\mathrm{P}$ & $\mathrm{P}$ & $\mathrm{P}$ & $\mathrm{P}$ & $\mathrm{P}$ & $\mathrm{P}$ & $\mathrm{P}$ & $\mathrm{P}$ & $\mathrm{P}$ & $\mathrm{P}$ & $\mathrm{P}$ & $\mathrm{P}$ & $\mathrm{P}$ & $\mathrm{P}$ & $P$ & $\mathrm{P}$ & $\mathrm{P}$ & $\mathrm{P}$ & $\mathrm{P}$ \\
\hline Pressão arterial & $\mathrm{P}$ & & $\mathrm{P}$ & $\mathrm{P}$ & $\mathrm{P}$ & $\mathrm{P}$ & $\mathrm{P}$ & $\mathrm{P}$ & $\mathrm{P}$ & $\mathrm{P}$ & $\mathrm{P}$ & $\mathrm{P}$ & $\mathrm{P}$ & $\mathrm{P}$ & $\mathrm{P}$ & $\mathrm{P}$ & $\mathrm{P}$ & $\mathrm{P}$ & $\mathrm{P}$ & $\mathrm{P}$ & $\mathrm{P}$ & $\mathrm{P}$ & $\mathrm{P}$ & $\mathrm{P}$ & $\mathrm{P}$ & $\mathrm{P}$ & $\mathrm{P}$ & $\mathrm{P}$ & $\mathrm{P}$ \\
\hline $\begin{array}{l}\text { Circunferência } \\
\text { da cintura }\end{array}$ & $\mathrm{P}$ & & $\mathrm{P}$ & $\mathrm{P}$ & & & $\mathrm{P}$ & $\mathrm{P}$ & $\mathrm{P}$ & $\mathrm{P}$ & $\mathrm{P}$ & $\mathrm{P}$ & $\mathrm{P}$ & $\mathrm{P}$ & $\mathrm{P}$ & $\mathrm{P}$ & $\mathrm{P}$ & $\mathrm{P}$ & $\mathrm{P}$ & $\mathrm{P}$ & $\mathrm{P}$ & $\mathrm{P}$ & $\mathrm{P}$ & $\mathrm{P}$ & $\mathrm{P}$ & $\mathrm{P}$ & $\mathrm{P}$ & $\mathrm{P}$ & $\mathrm{P}$ \\
\hline $\begin{array}{l}\text { Realização de } \\
\text { atividades físicas }\end{array}$ & $\mathrm{P}$ & $\mathrm{P}$ & & & & & $\mathrm{P}$ & $\mathrm{P}$ & & $\mathrm{P}$ & & $\mathrm{P}$ & $\mathrm{P}$ & $\mathrm{P}$ & $\mathrm{P}$ & & & $\mathrm{P}$ & & & & $\mathrm{P}$ & & & & $\mathrm{P}$ & & & \\
\hline Acidentes & $\mathrm{P}$ & $\mathrm{P}$ & & & $\mathrm{P}$ & $\mathrm{P}$ & $\mathrm{P}$ & & $\mathrm{P}$ & $\mathrm{P}$ & $\mathrm{P}$ & $\mathrm{P}$ & & & & & & & & & & & & & & & & & \\
\hline Diabetes & $\mathrm{P}$ & $\mathrm{P}$ & $\mathrm{P}$ & $\mathrm{P}$ & & & & $\mathrm{P}$ & $\mathrm{P}$ & $\mathrm{P}$ & & & $\mathrm{P}$ & $\mathrm{P}$ & $\mathrm{P}$ & $\mathrm{P}$ & & & $\mathrm{P}$ & $\mathrm{P}$ & $\mathrm{P}$ & $\mathrm{P}$ & $\mathrm{P}$ & $\mathrm{P}$ & $P$ & $\mathrm{P}$ & $\mathrm{P}$ & $\mathrm{P}$ & $\mathrm{P}$ \\
\hline Asma & $\mathrm{P}$ & $\mathrm{P}$ & & & $\mathrm{P}$ & $\mathrm{P}$ & $\mathrm{P}$ & $\mathrm{P}$ & $\mathrm{P}$ & & $\mathrm{P}$ & $\mathrm{P}$ & & $\mathrm{P}$ & & & & & & & & & & & & & & $\mathrm{P}$ & \\
\hline $\begin{array}{l}\text { Hipertensão } \\
\text { arterial }\end{array}$ & $\mathrm{P}$ & $\mathrm{P}$ & & $\mathrm{P}$ & & & & $\mathrm{P}$ & $\mathrm{P}$ & $\mathrm{P}$ & & & $\mathrm{P}$ & $\mathrm{P}$ & $\mathrm{P}$ & $\mathrm{P}$ & & & $\mathrm{P}$ & $\mathrm{P}$ & $\mathrm{P}$ & $\mathrm{P}$ & $\mathrm{P}$ & $\mathrm{P}$ & $\mathrm{P}$ & $\mathrm{P}$ & $\mathrm{P}$ & $\mathrm{P}$ & $\mathrm{P}$ \\
\hline Colesterol & $\mathrm{P}$ & $\mathrm{P}$ & & & & & & $\mathrm{P}$ & $\mathrm{P}$ & $\mathrm{P}$ & & & $\mathrm{P}$ & $\mathrm{P}$ & $\mathrm{P}$ & $\mathrm{P}$ & & $\mathrm{P}$ & $\mathrm{P}$ & $\mathrm{P}$ & $\mathrm{P}$ & $\mathrm{P}$ & $\mathrm{P}$ & $\mathrm{P}$ & $\mathrm{P}$ & $\mathrm{P}$ & $\mathrm{P}$ & $\mathrm{P}$ & $\mathrm{P}$ \\
\hline Problemas renais & $\mathrm{P}$ & $\mathrm{P}$ & & & & & & & & & & & & & & & & & $\mathrm{P}$ & $\mathrm{P}$ & & & & & & & & & \\
\hline Saúde materna & $\mathrm{P}$ & $\mathrm{P}$ & & & & & & & & & & $\mathrm{P}$ & & & & & & & & & & & & & & & & & \\
\hline $\begin{array}{l}\text { Problemas } \\
\text { respiratórios }\end{array}$ & $\mathrm{P}$ & $\mathrm{P}$ & $\mathrm{P}$ & $\mathrm{P}$ & & & & $\mathrm{P}$ & & $\mathrm{P}$ & & & & & & & & & & & & & & & & & & & \\
\hline Saúde bucal & $\mathrm{P}$ & $\mathrm{P}$ & & & & & & & $\mathrm{P}$ & $\mathrm{P}$ & $\mathrm{P}$ & $\mathrm{P}$ & & & $\mathrm{P}$ & & & & & $\mathrm{P}$ & $\mathrm{P}$ & & & & & & & & $\mathrm{P}$ \\
\hline Plano de saúde & $\mathrm{P}$ & $\mathrm{P}$ & & & & & & & & & & & & & & & & & $\mathrm{P}$ & $\mathrm{P}$ & $\mathrm{P}$ & $\mathrm{P}$ & & $\mathrm{P}$ & $\mathrm{P}$ & $\mathrm{P}$ & & $\mathrm{P}$ & \\
\hline Contracepção & $\mathrm{P}$ & $\mathrm{P}$ & $\mathrm{P}$ & $\mathrm{P}$ & $\mathrm{P}$ & & $\mathrm{P}$ & $\mathrm{P}$ & $\mathrm{P}$ & & $\mathrm{P}$ & $\mathrm{P}$ & $\mathrm{P}$ & $\mathrm{P}$ & $\mathrm{P}$ & $\mathrm{P}$ & & & & $\mathrm{P}$ & & & & & & & & & \\
\hline $\begin{array}{l}\text { Problemas na } \\
\text { alimentação }\end{array}$ & $\mathrm{P}$ & $\mathrm{P}$ & & & & & & & & & & & & & & & & & & & & & & & & & & & $\mathrm{P}$ \\
\hline Saúde mental & $\mathrm{P}$ & $\mathrm{P}$ & & & & & & & & & & & & & & & & & & & & $\mathrm{P}$ & & $\mathrm{P}$ & $\mathrm{P}$ & $\mathrm{P}$ & & & \\
\hline $\begin{array}{l}\text { Reposição } \\
\text { hormonal }\end{array}$ & $\mathrm{P}$ & $\mathrm{P}$ & & & & & & $\mathrm{P}$ & $\mathrm{P}$ & & $\mathrm{P}$ & $\mathrm{P}$ & $\mathrm{P}$ & $\mathrm{P}$ & $\mathrm{P}$ & $\mathrm{P}$ & & & & & & & & & & & & & \\
\hline Atividade sexual & & $\mathrm{P}$ & & & & & & & & & & & & & & & & & & $\mathrm{P}$ & & $\mathrm{P}$ & & & & & & & \\
\hline $\begin{array}{l}\text { Orientação } \\
\text { sexual }\end{array}$ & & $\mathrm{P}$ & & & & & & & & & & & & & & & & & & & $\mathrm{P}$ & $\mathrm{P}$ & $\mathrm{P}$ & $\mathrm{P}$ & $\mathrm{P}$ & $\mathrm{P}$ & $\mathrm{P}$ & $\mathrm{P}$ & $\mathrm{P}$ \\
\hline Vacinação & $\mathrm{P}$ & $\mathrm{P}$ & & & & & & & & & $\mathrm{P}$ & $\mathrm{P}$ & $\mathrm{P}$ & $\mathrm{P}$ & $\mathrm{P}$ & $\mathrm{P}$ & $\mathrm{P}$ & $\mathrm{P}$ & & & & & & & & & & & \\
\hline $\begin{array}{l}\text { Uso de } \\
\text { medicamento }\end{array}$ & $\mathrm{P}$ & $\mathrm{P}$ & $\mathrm{P}$ & $\mathrm{P}$ & $\mathrm{P}$ & $\mathrm{P}$ & $\mathrm{P}$ & $\mathrm{P}$ & $\mathrm{P}$ & $\mathrm{P}$ & $\mathrm{P}$ & $\mathrm{P}$ & $\mathrm{P}$ & $P$ & $\mathrm{P}$ & $\mathrm{P}$ & $\mathrm{P}$ & $\mathrm{P}$ & $\mathrm{P}$ & $\mathrm{P}$ & $\mathrm{P}$ & $\mathrm{P}$ & $\mathrm{P}$ & $\mathrm{P}$ & $\mathrm{P}$ & $\mathrm{P}$ & $\mathrm{P}$ & $\mathrm{P}$ & $\mathrm{P}$ \\
\hline $\begin{array}{l}\text { Deficiência } \\
\text { auditiva }\end{array}$ & & $\mathrm{P}$ & & & & & & & & & & & & & & & & & & & & & & $\mathrm{P}$ & & & & & \\
\hline
\end{tabular}

Fontes e informações adicionais: ${ }^{\mathrm{a}} 41,42,43$.

b 46. Não foi localizado o histórico de temas abordados na HSE nos anos de 1991 e 1992. 
cipais problemas de saúde vivenciados por cada país. Desse modo, análises comparativas são factíveis e desejáveis, entretanto, qualquer tema a ser investigado demanda o estudo aprofundado das especificidades das pesquisas, contextos e trajetórias socioeconômicas dos dois países diante do objeto de estudo selecionado.

\section{Considerações finais}

A estruturação de sistemas de informação em saúde é fundamental para o acompanhamento das condições de vida e bem estar da população. Tais sistemas incluem múltiplas pesquisas e registros, com caráter amostral e censitário. No caso da Inglaterra, o Censo Demográfico traz questões sobre as condições gerais de saúde e, no Brasil, sobre deficiências. Enquanto os Censos Demográficos em ambos os países ocorrem a cada dez anos, a periodicidade das pesquisas amostrais voltadas à compreensão da saúde da população varia enormemente no Brasil e Inglaterra, como se evidenciou no caso da PNS e HSE apresentadas neste artigo. Tal diferença de periodicidade, entretanto, não impede comparações entre os países. Em realidade, o conjunto de temas comuns investigados nos anos recentes em ambas as pesquisas abre um leque de múltiplas possibilidades investigativas para pensar similitudes e diferenças entre os contextos latino-americano e europeu.

No Brasil, a análise da PNS 2013 permitiu, por exemplo, mostrar diferenças no uso dos serviços de saúde por portadores de doenças crônicas, níveis de renda e planos de saúde privados ${ }^{47}$. $\mathrm{Na}$ Inglaterra, com base na HSE foi possível inferir que houve, a partir da década de 1990, aumento da prevalência de condições de saúde auto -reportadas como ruins e de diagnósticos clínicos de diabetes e sobrepeso nas coortes mais jovens, com crescimento dos anos vividos com presença de morbidades e os ganhos da expectativa de vida saudável foram menores em relação aos da expectativa de vida ${ }^{48}$. Os usos da HSE também ilustram a importância do pareamento de dados em pesquisas de saúde, o que seria um potencial para a PNS. No caso, a HSE tem os dados pareados com as informações de registros mortalidade e câncer, possibilitando análises longitudinais e de coorte a partir de uma pesquisa transversal ${ }^{49}$.

Vale lembrar que comparações entre condições de saúde no Brasil e na Inglaterra não são aleatórias. Muito pelo contrário: ambos os países têm sistemas de saúde bastante robustos, gratuitos e de cobertura universal, isto é, abarcando virtualmente todos os membros de suas respectivas sociedades. Entretanto, a despeito das particularidades do National Health Service (NHS) inglês e do Sistema Único de Saúde (SUS) brasileiro, ambos têm em comum o contexto de crises de financiamento cada vez mais agudas, que repercutem sobre a qualidade do serviço oferecido à população, assim como dificultam o acesso, especialmente dos segmentos populacionais em maior vulnerabilidade social, que historicamente já apresentam os piores indicadores de saúde.

A PNS e a HSE apresentam excelentes oportunidades para aferir a dinâmica do perfil de morbimortalidade das populações do Brasil e da Inglaterra, tanto separadamente quanto em estudos comparativos. Esse acompanhamento é de fundamental importância para lançar luz sobre os determinantes sociais de saúde nos dois países, permitindo o aprofundamento do conhecimento científico assim como a elaboração de políticas públicas focalizadas nos segmentos populacionais mais vulneráveis. Avança-se, dessa forma, na direção da garantia do acesso à saúde com equidade, além da construção paulatina de um desenvolvimento social inclusivo.

\section{Colaboradores}

Todos os autores trabalharam igualmente na concepção, redação e análise dos dados do artigo. 


\section{Referências}

1. Malta DC, Leal MC, Costa MFL, Morais Neto OL. In quéritos Nacionais de Saúde: experiência acumulada e proposta para o inquérito de saúde brasileiro. Rev Bras Epidemiol 2008; 11(Supl. 1):159-167.

2. Dyson T. The role of the demographic transition in the process of urbanization. Pop Dev Review 2011; 37(Supl. 1):34-54.

3. Chesnais JC. The Demographic transition: stages, patterns, and economic implications - a longitudinal study of sixty-seven countries covering the period 1720-1984. Oxford: Clarendon Press; 1992.

4. Vasconcelos AMN, Gomes MMF. Transição demográfica: a experiência brasileira. Epidemiol Serv Saúde 2012; 21(4):539-548.

5. Wong L, Carvalho JAM. O rápido processo de envelhecimento populacional do Brasil: sérios desafios para as políticas públicas. $R$ Bras Est Pop 2006; 23(1):5-26.

6. Brito F. Transição demográfica e desigualdades sociais no Brasil. $R$ Bras Est Pop 2008; 25(1):5-26.

7. Carmo RL, Camargo K. Dinâmica demográfica brasileira recente: padrões regionais de diferenciação. Texto para discussão IPEA 2415, Brasília: Rio de Janeiro, Ipea; 2018.

8. Woods R. The demography of Victorian England and Wales. Cambridge, UK: Cambridge University Press; 2000.

9. Jaadla H, Reid A, Garret, E, Schurer, E, Day J. Revisiting the Fertility Transition in England and Wales: The Role of Social Class and Migration. Demography 2020; 57:1543-1569.

10. Coale AJ, Watkins SC. The decline of fertility in Europe. Princeton: Princeton University Press; 2017.

11. Office for National Statistics. Births in England and Wales: 2019. Londres, Reino Unido; 2020. [acessado 2021 jan 12]. Disponível em: https://bit.ly/2L9fgwg

12. Office for National Statistics. Deaths Registered in England and Wales 2015. Londres, Reino Unido; 2016. [acessado 2021 jan 12]. Disponível em: https://bit. ly/2MA10gi

13. Szreter S. Fertility, social class, gender, and the professional model: statistical explanation and historical significance. Econ hist rev 2015; 68(2):707-722.

14. Mckeown T. The modern rise of population. London: Edward Arnold; 1976.

15. Harris B, Hinde A. Sanitary investment and the decline of urban mortality in England and Wales, 18171914. The history of the Family 2019; 24(2):339-376.

16. Leon DA, Jdanov DA, Shkolnikov VM. Trends in life expectancy and age-specific mortality in England and Wales, 1970-2016, in comparison with a set of 22 high -income countries: an analysis of vital statistics data. Lancet Public Health 2019; 4: 575-582.

17. Hiam L, Dorling D, Mckee M. Things Fall Apart: the British Health Crisis 2010-2020. British Medical Bulletin 2020; 133(1):4-15.

18. Taylor-Robinson D, Lai ETC, Wickham S, Rose T, Norman P, Bambra C, Whitehead M, Barr B. Assessing the impact of rising child poverty on the unprecedented rise in infant mortality in England, 2000-2017: time trend analysis. BMJ Open 2019; 9(10):1-6.
19. Joyce $\mathrm{R}, \mathrm{Xu} \mathrm{X}$. Inequalities in the twenty-first century. The Institute for Fiscal Studies; 2019. [acessado 2021 jan 12]. Disponível em: https://bit.ly/3orPUHv

20. Bennet JE, Pearson-Stuttard J, Kontis V, Capewell S, Wolfe I, Ezzati M. Contributions of diseases and injuries to widening life expectancy inequalities in England from 2001 to 2016: a population-based analysis of vital registration data. The Lancet Public Health 2018; 3(12):586-597.

21. Omram AR. The epidemiologic transition: a theory of the epidemiology of population change. Milbank Memorial Fund Quarterly 1971; (49)1:509-538.

22. Prata PR. A transição epidemiológica no Brasil. Cad Saude Publica 1992; 8(2):168-175.

23. Araújo JD. Polarização epidemiológica no Brasil. Epidemiol Serv Saude 2012;21(4):533-538.

24. Duarte EC, Barreto SM. Transição demográfica e epidemiológica: a Epidemiologia e Serviços de Saúde revisita e atualiza o tema. Epidemiol Serv Saude 2012; 21(4):529-532.

25. Schramm JMA, Oliveira AF, Leite IC, Valente JG, Gadelha AMJ, Portela MC, Campos MR. Transição epidemiológica e o estudo de carga de doença no Brasil. Cien Saude Colet 2004; 9(4):897-908.

26. Paes-Sousa R, Schramm JMA, Mendes, LVP. Fiscal austerity and the health sector: the cost of adjustments. Cien Saude Colet 2019; 24(12):4375-4384.

27. Hone T, Mirelman, AJ, Rasella D, Paes-Sousa R, Barreto ML, Rocha R, Millett C. Effect of economic recession and impact of health and social protection expenditures on adult mortality: a longitudinal analysis of 5565 Brazilian municipalities. Lancet Global Health 2019; 7:1575-1583.

28. Instituto Brasileiro de Geografia e Estatística (IBGE). Estimativas da População - 2019. Rio de Janeiro: IBGE; 2019. [acessado 2021 jan 15]. Disponível em: https:// bit.ly/3r1X5HU

29. Office for National Statistics. Estimates of the population for the UK, England and Wales, Scotland and Northern Ireland. Londres: Office for National Statistics; 2019. [acessado 2021 Jan 15]. Disponível em: https:// bit.ly/3t0t5hD

30. Banco Mundial. Gini Index. Washington (EUA): Banco Mundial; 2021a. [acessado 2021 Jan 15]. Disponível em: https://bit.ly/2Yv5UO9

31. Banco Mundial. World bank country and lending groups. Washington (EUA): Banco Mundial; 2021b. [acessado 2021 Jan 15]. Disponível em: https://bit. ly/2KWDk5e

32. Banco Mundial. Reversals of fortune: Prosperity and shared prosperity 2020. Washington (EUA): Banco Mundial; 2020. [acessado 2021 Jan 15]. Disponível em: https://bit.ly/2Yv66gl

33. Organização das Nações Unidas (ONU). World Urbanization Prospects. Nova Iorque (EUA): ONU; 2018. [acessado 2021 Jan 15]. Disponível em: https://bit. ly/3pvL3Gt

34. Organização das Nações Unidas (ONU). World Population Prospects. Nova Iorque (EUA): ONU; 2018 [acessado 2021 Jan 15]. Disponível em: https://bit. ly/3abJJ5k 
35. Banco Mundial. Mortality rate, under-5 per 1,000 live births. Washington (EUA): Banco Mundial; 2021c. [acessado 2021 Jan 15]. Disponível em: https://bit. ly/2NAloyf

36. Banco Mundial. People using safely managed sanitation services (\% of population). Washington (EUA): Banco Mundial; 2021d. [acessado 2021 Jan 15]. Disponível em: https://bit.ly/39scFqA

37. Global Health Data. Country profiles. Seattle (EUA): Global Health Data; 2021 [acessado 2021 Jan 15]. Disponível em: https://bit.ly/3acy5H9

38. Global Health Data. Deaths rates by cause. Seattle (EUA): Global Health Data; 2021 [acessado 2021 Jan 15]. Disponível em: https://bit.ly/39sw0aV

39. Machado FSN, Carvalho, MAP, Mataresi, A, Mendonça ET, Cardoso LM, Yogi, MS, Rigato HM, Salazar M. Utilização da telemedicina como estratégia de promoção de saúde em comunidades ribeirinhas da Amazônia: experiência de trabalho interdisciplinar, integrando as diretrizes do SUS. Cien Saude Colet 2010; 15(1):247-254.

40. Travassos C, Viacava F, Laguardia J. Os Suplementos Saúde na Pesquisa Nacional por Amostra de Domicílios (PNAD) no Brasil. Rev Bras Epidemiol 2008; 11(s1):98-112.

41. Damacena GN, Szwarcwald CL, Malta DC, Souza Junior PRB, Vieira MLFP, Pereira CA, Neto OLM, Silva Júnior JB. O processo de desenvolvimento da Pesquisa Nacional de Saúde no Brasil, 2013. Epidemiol Serv Saude 2015; 24:197-206.

42. Szwarcwald CL, Malta DC, Pereira CA, Vieira MLFP, Conde WL, Souza Junior PRB, Damacena GN, Azevedo LO, Silva GA, Filha MMT, Lopes CS, Romero DE, Almeida WS, Monteiro CA. Pesquisa Nacional de Saúde no Brasil: concepção e metodologia de aplicação. Cien Saude Colet 2014; 19:333-342.

43. Instituto Brasileiro de Geografia e Estatística (IBGE). Pesquisa nacional de saúde 2019: percepção do estado de saúde, estilos de vida, doenças crônicas e saúde bucal: Brasil e grandes regiões. Rio de Janeiro: IBGE; 2020.
44. Souza-Junior PRB, Freitas MPS, Antonaci GA, Szwarcwald CL. Desenho da amostra da Pesquisa Nacional de Saúde 2013. Epidemiol Serv Saude 2015; 24(2):207216.

45. Health and Social Care Information Centre. Health Survey for England 2019 - Methods. Londres: Health and Social Care Information Centre; 2020. [acessado 2021 Jan 15]. Disponível em: https://bit.ly/36iDLyg

46. National Health Service (NHS). Health Survey for England Content. Londres NHS; 2021. [acessado 2021 Jan 12]. Disponível em: https://bit.ly/2YrqfnA

47. Malta DC, Bernal RTI, Lima MG, Araújo SSC, Silva MMA, Freitas MIF, Barros MBA. Doenças crônicas não transmissíveis e a utilização de serviços de saúde: análise da Pesquisa Nacional de Saúde no Brasil. Rev Saude Publica 2017; 51(S1)4s.

48. Jivraj S, Alissa G, Benedetta P, George BP. Living longer but not necessarily healthier: the joint progress of health and mortality in the working-age population of England. Pop Studies 2020; 74(3):399-414.

49. Mindell J, Biddulph JP, Hirani V, Stamatakis E, Craig R, Nunn S, Shelton N. Cohort profile: the health survey for England. Int J Epidemiol 2012; 41(6):15851593.

Artigo apresentado em 02/02/2021

Aprovado em 02/02/2021

Versão final apresentada em 04/02/2021

Editores-chefes: Maria Cecília de Souza Minayo, Romeu Gomes, Antônio Augusto Moura da Silva 